\title{
Transcriptomics Data Integration Reveals Jak-STAT as a Common Pathway Affected by Pathogenic Intracellular Bacteria in Natural Reservoir Hosts
}

Ruth C. Galindo ${ }^{1}$ and José de la Fuente ${ }^{1,2 *}$

${ }^{1}$ Instituto de Investigación en Recursos Cinegéticos IREC-CSIC-UCLM-JCCM, Ronda de Toledo s/n, 13005 Ciudad Real, Spain

${ }^{2}$ Department of Veterinary Pathobiology, Center for Veterinary Health Sciences, Oklahoma State University, Stillwater, OK 74078, USA

\begin{abstract}
The study of the host-pathogen interface in natural reservoir hosts is essential to identify host-cell mechanisms affected by bacterial infection and persistence. Herein we used the Database for Annotation, Visualization and Integrated Discovery (DAVID) to integrate transcriptomics data and find common host-cell biological processes, molecular functions and pathways affected by pathogenic intracellular bacteria of the genera Anaplasma, Brucella and Mycobacterium during infection and persistence in two natural reservoir hosts, wild boar and sheep. The results showed that the upregulation of host innate immune pro-inflammatory genes and signaling pathways constitutes a general antibacterial mechanism in response to intracellular bacteria. Pathway focused analysis revealed a role for the Jak-STAT pathway during bacterial intracellular infection, a fact reported before in Mycobacterium infected cells but not during Brucella spp. and A. phagocytophilum infection. A clear activation of the Jak-STAT pathway was observed in A. phagocytophilum infected wild boar and sheep when compared to uninfected controls. Brucella spp. infection resulted in a balanced regulation of the Jak-STAT signaling and $M$. bovis infection of wild boar clearly produced a downregulation of some of the Jak-STAT effectors such as IL5 and TKY2. These results suggested that mycobacteria and brucellae induce host innate immune responses while manipulating adaptive immunity to circumvent host-cell defenses and establish infection. In contrast, $A$. phagocytophilum infection induces both innate and adaptive immunity, those suggesting that this pathogen uses other mechanisms to circumvent host-cell defenses by downregulating other adaptive immune genes and delaying the apoptotic death of neutrophils through activation of the Jak-STAT pathway among other mechanisms.
\end{abstract}

Keywords: Transcriptomics; Anaplasma; Mycobacterium; Brucella; Systems biology

\section{Introduction}

Pathogenic bacteria have to interact with host cells and reprogram the complex molecular and cellular networks of these cells to allow bacterial infection, replication and spread, while countering hostdefense mechanisms [1,2]. This process is likely to involve genes from both pathogens and hosts, all of which are probably subject to complex regulation [1-3].

Molecular biology and in particular recent advances in genomics, transcriptomics and proteomics have allowed the characterization of host-pathogen interactions [2,3]. However, these studies have focused on the response of particular hosts to one or multiple pathogens, mostly using in vitro systems (see for example, [2]). Moving from in vitro studies in cultured cells to relevant animal disease models and natural reservoir hosts is crucial for understanding host-pathogen interactions, yet such studies are often neglected because cell culture-based systems are easier to manipulate. However, the study of the host-pathogen interface in natural reservoir hosts infected with different pathogens is now possible and essential to identify host-cell mechanisms affected by bacterial infection and persistence, which may be different from those identified in vitro $[3,4]$.

Herein, the Database for Annotation, Visualization and Integrated Discovery (DAVID) was used to integrate transcriptomics data and find common host-cell biological processes, molecular functions and pathways affected by pathogenic intracellular bacteria of the genera Anaplasma, Brucella and Mycobacterium during infection and persistence in two natural reservoir hosts, wild boar (Sus scrofa) and sheep (Ovis aries).

\section{Materials and Methods}

\section{Transcriptomics data}

Transcriptomics data was obtained from previously published studies on infected and uninfected matching control animals using microarray hybridization and real-time RT-PCR in wild boar infected with Mycobacterium bovis, Anaplasma phagocytophilum and Brucella suis [4-6] (NCBI Gene Expression Omnibus (GEO) platform accession and series numbers GPL3533, GPL3533, GSE15766, GSE17492) and in sheep infected with A. phagocytophilum and Brucella ovis [7-9] (GPL4456, GPL6954, GSE11928 and GSE10286). In these studies, the infection with M. bovis, A. phagocytophilum, B. ovis or B. suis strains was characterized in experimentally or naturally infected animals during acute or chronic infection (Table 1).

\section{Transcriptomics data integration and analysis}

Microarray data from all host-pathogen interactions were filtered to select significant $(\mathrm{P}<0.05)$ differentially expressed genes with an infected/uninfected fold change $(\mathrm{FC}) \geq 1.2$. These genes were analyzed using DAVID V6.7 (http://david.abcc.ncifcrf.gov/) [10,11] to select

*Corresponding author: José de la Fuente, Instituto de Investigación en Recursos Cinegéticos IREC-CSIC-UCLM-JCCM, Ronda de Toledo s/n, 13005 Ciudad Real, Spain, Tel: 34-926295450; Fax: 34-926295451; E-mail: jose_delafuente@yahoo.com

Received March 07, 2012; Accepted April 17, 2012; Published April 30, 2012

Citation: Galindo RC, de la Fuente J (2012) Transcriptomics Data Integration Reveals Jak-STAT as a Common Pathway Affected by Pathogenic Intracellular Bacteria in Natural Reservoir Hosts. J Proteomics Bioinform 5: 108-115. doi:10.4172/jpb.100022

Copyright: (c) 2012 Galindo RC, et al. This is an open-access article distributed under the terms of the Creative Commons Attribution License, which permits unrestricted use, distribution, and reproduction in any medium, provided the original author and source are credited. 
Citation: Galindo RC, de la Fuente J (2012) Transcriptomics Data Integration Reveals Jak-STAT as a Common Pathway Affected by Pathogenic Intracellular Bacteria in Natural Reservoir Hosts. J Proteomics Bioinform 5: 108-115. doi:10.4172/jpb.1000221

the highest enrichment score (ES), which is the geometric mean of all enrichment $P$ values (EASE scores) for each gene ontology (GO) term [11] and clustering for host cell biological processes, molecular functions and pathways affected by bacterial infection in these hosts and then identify among them those factors common to all host-bacteria interactions (Figure 1). The significance of GO term enrichment was determined with a modified Fisher's exact test (EASE score; $\mathrm{P} \leq$ 0.001 ) and a FC $>2$ for overrepresented terms. Enrichment P-values were globally corrected to control family-wide false discovery rates at Benjamini $\leq 0.0004$. ES $>2$ was used to rank GO term enrichment.

\section{Real-time reverse transcription (RT)-PCR}

Differential expression of genes in common host-cell biological processes, molecular functions and pathways affected by pathogenic intracellular bacteria was analyzed by real-time RT-PCR using primers designed based on sequences available in the GenBank (Table 2). The real-time RT-PCR was performed on pooled RNA samples from infected and uninfected wild boar and sheep (wild boar infected with A. phagocytophilum, $\mathrm{N}=2$; wild boar infected with Brucella spp., $\mathrm{N}=3$; wild boar infected with $M$. bovis, $\mathrm{N}=6$; sheep infected with $A$. phagocytophilum, $\mathrm{N}=2$; sheep infected with Brucella spp, $\mathrm{N}=6$; wild boar uninfected controls, $\mathrm{N}=12$; sheep uninfected controls, $\mathrm{N}=5$ ) with gene specific primers using the iScript One-Step RT-PCR Kit with SYBR Green and the iQ5 thermal cycler (Bio-Rad, Hercules, CA, USA) following manufacturer's recommendations. The mRNA levels were normalized against cyclophlilyn and beta-actin using the genNorm method (ddCT method as implemented by Bio-Rad iQ5 Standard Edition, Version 2.0) [12]. In all cases, the mean of triplicate values was used and data from infected and uninfected animals were compared using the Student's t-test $(\mathrm{P}=0.05)$. Correlation analysis between microarray and RT-PCR results was conducted in Excel by calculating (a) percent of values with similar tendency (i.e. no variation, upregulated or downregulated) and (b) correlation coefficients $\left(\mathrm{R}^{2}\right)$ between all values independently of the statistical analysis for RT-PCR results which were affected by the low number of samples used in the analysis.

\section{Results and Discussion}

The analysis conducted here focused on wild boar infected with M. bovis, A. phagocytophilum and B. suis [4-6] and sheep infected with A. phagocytophilum and B. ovis [7-9]. These pathogens represent intracellular bacteria that infect and replicate within host immune cells and were selected because of their impact as zoonotic pathogens in many regions of the world.

An analysis pipeline was developed using DAVID to integrate data and find common host-cell biological processes, molecular functions and pathways affected by pathogenic intracellular bacteria of the genera Anaplasma, Brucella and Mycobacterium during infection and persistence in two natural reservoir hosts, Eurasian wild boar and sheep (Figure 1). Because transcriptomics data were obtained from different experiments with tissue samples collected at different infection times and conditions [4-9] (Table 1), differences between various hostpathogen interactions could be explained by different factors. These factors include differences in the transcriptomics methods employed (microarray and data analysis platforms), experimental conditions (natural or experimental infections), host tissues used for RNA extraction (peripheral blood mononuclear cells (PBMC) or spleen) and individual variability of both pathogens and hosts. However, we hypothesized that statistically significant common factors emerging despite all these differences, have a particular relevance in identifying host-pathogen interactions of different pathogenic intracellular bacteria in different hosts, thus allowing the identification of common mechanisms that may be used for infection characterization, control and prevention. Therefore, the analysis focused on common mechanisms affected by these bacteria in all host-pathogen interactions.

\section{Common host-cell biological processes, molecular functions and pathways affected by Anaplasma, Brucella and Mycobacterium infection in wild boar and sheep}

The results showed that it is possible to integrate data from different trascriptomics experiments to find common mechanisms affected by pathogenic intracellular bacteria in natural reservoir hosts. Common

\begin{tabular}{|c|c|c|c|c|c|}
\hline Strain & Origin & Host & $\begin{array}{l}\text { Tissue } \\
\text { examined }\end{array}$ & Infection type & Characterization of infection \\
\hline \multirow[t]{2}{*}{ A. phagocytophilum } & $\begin{array}{l}\text { Isolated from infected sheep in the Basque } \\
\text { Country, Spain [30] (Genbank accession } \\
\text { number EU436164) }\end{array}$ & Sheep & PBMC & $\begin{array}{l}\text { Experimental } \\
\text { acute infection }\end{array}$ & $\begin{array}{l}\text { Infection was confirmed by microscopic } \\
\text { examination of stained blood smears and } m s p 4 \\
\text { PCR }[7,30]\end{array}$ \\
\hline & $\begin{array}{l}\text { Isolated from infected Eurasian wild boar } \\
\text { hunter-killed in Slovenia, genotipically } \\
\text { identical to strains isolated from humans, } \\
\text { dogs and I. ricinus ticks [31] (Genbank } \\
\text { accession numbers AY055469, AF033101 } \\
\text { and EU246961) }\end{array}$ & $\begin{array}{l}\text { Wild } \\
\text { boar }\end{array}$ & PBMC & $\begin{array}{l}\text { Natural chronic } \\
\text { infection }\end{array}$ & $\begin{array}{l}\text { Infection was confirmed by } 16 S \text { rDNA and } \\
\text { groESL PCRs and sequence analysis [31] }\end{array}$ \\
\hline $\begin{array}{l}\text { B. ovis } \\
R \text { virulent PA strain }\end{array}$ & $\begin{array}{l}\text { Provided by Dr. J.M. Verger. Unite' } \\
\text { d'Infectiologie Animale et Sante' Publique, } \\
\text { INRA, Nouzilly, France }[32,33]\end{array}$ & Sheep & PBMC & $\begin{array}{l}\text { Experimental } \\
\text { acute infection }\end{array}$ & $\begin{array}{l}\text { Infection was confirmed at necropsy by bacterial } \\
\text { culture, morphology, Gram staining, oxidase } \\
\text { and urease tests, } \mathrm{CO}_{2} \text { requirements and phage } \\
\text { typing }[8,34]\end{array}$ \\
\hline $\begin{array}{l}\text { B. suis } \\
\text { biovar } 2\end{array}$ & $\begin{array}{l}\text { Isolated from infected Eurasian wild boar in } \\
\text { Navarra, Spain }[34,35]\end{array}$ & $\begin{array}{l}\text { Wild } \\
\text { boar }\end{array}$ & Spleen & $\begin{array}{l}\text { Natural chronic } \\
\text { infection }\end{array}$ & $\begin{array}{l}\text { Infection was confirmed by bacterial culture and } \\
\text { seroconversion }[5,36]\end{array}$ \\
\hline M. bovis & $\begin{array}{l}\text { Isolated from infected Eurasian wild boar in } \\
\text { Southwestern Spain [3] }\end{array}$ & $\begin{array}{l}\text { Wild } \\
\text { boar }\end{array}$ & Spleen & $\begin{array}{l}\text { Natural chronic } \\
\text { infection }\end{array}$ & $\begin{array}{l}\text { Infection was confirmed at necropsy by } \\
\text { pathology, bacterial culture and spoligotyping } \\
{[3,4]}\end{array}$ \\
\hline
\end{tabular}

Table 1: Bacterial strains and experimental animals. 
Citation: Galindo RC, de la Fuente J (2012) Transcriptomics Data Integration Reveals Jak-STAT as a Common Pathway Affected by Pathogenic Intracellular Bacteria in Natural Reservoir Hosts. J Proteomics Bioinform 5: 108-115. doi:10.4172/jpb.1000221

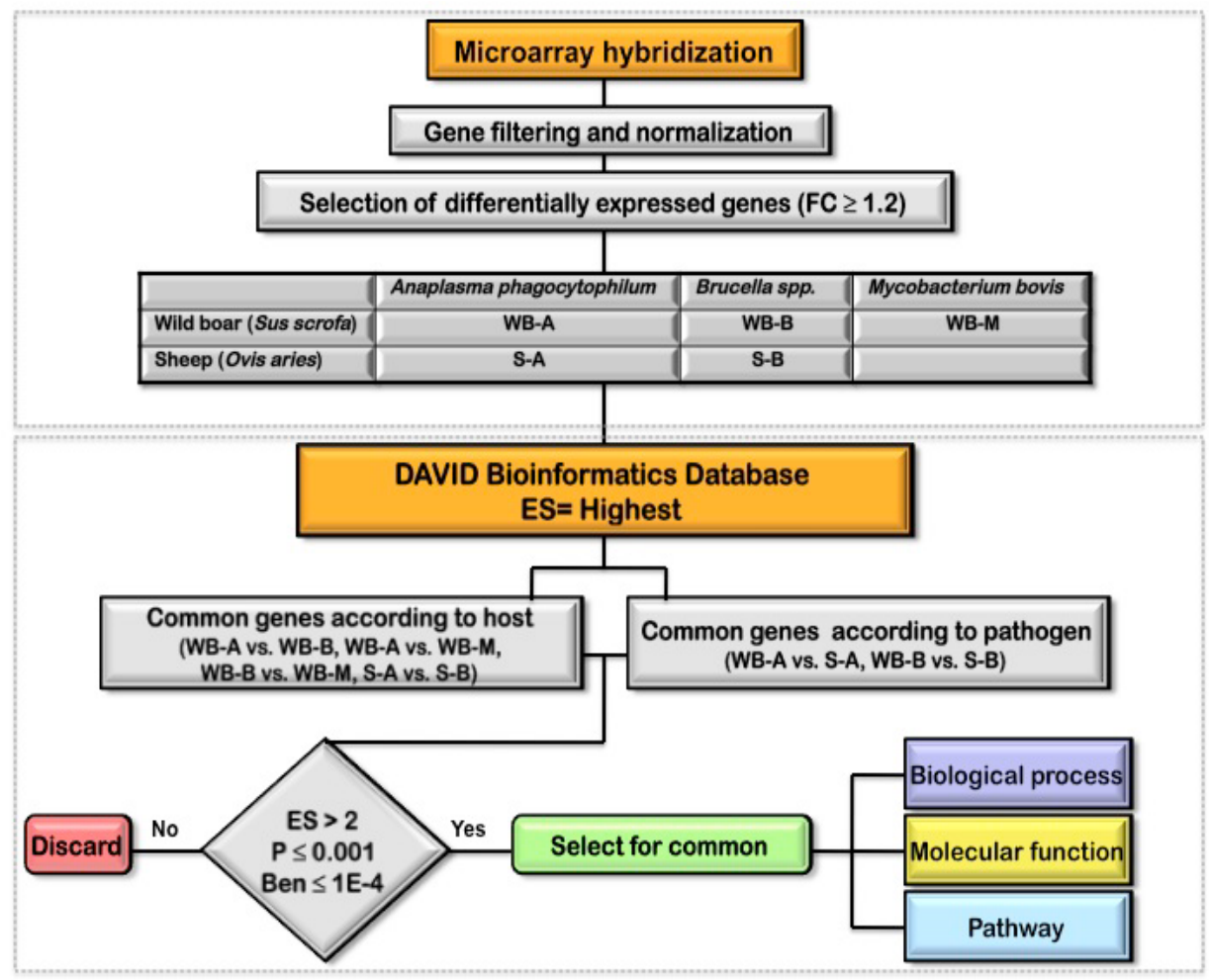

Figure 1: Analysis pipeline. The analysis used the Database for Annotation, Visualization and Integrated Discovery (DAVID) V 6.7 (http://david.abcc.ncifcrf.gov/) to integrate data and find common host-cell biological processes, molecular functions and pathways affected by pathogenic intracellular bacteria of the genera Anaplasma, Brucella and Mycobacterium during infection in natural reservoir hosts, wild boar and sheep. Abbreviations: ES, enrichment score; FC, fold change; Ben, Benjamini; WBA, wild boar infected with A. phagocytophilum; WB-B, wild boar infected with B. suis; WB-M, wild boar infected with $M$. bovis; S-A, sheep infected with A. phagocytophilum; $\mathrm{S}-\mathrm{B}$, sheep infected with $B$. ovis.

host-cell biological processes affected by Anaplasma, Brucella and Mycobacterium infection in wild boar and sheep included regulation of immune system and immune system with 33 genes represented (Tables 3 and 4). The common host-cell molecular functions affected included 28 genes with receptor binding, cytokine activity and growth factor activity (Tables 3 and 4). The common host-cell pathways affected by these bacteria were cytokine-receptor interaction, hematopoietic cell lineage and Janus Kinase-Signal Transducer and Activator of Transcription (Jak-STAT) signaling pathway (Table 3). A good correlation was obtained between microarray and RT-PCR results for genes in common host-cell biological processes, molecular functions and pathways affected by pathogenic intracellular bacteria (Table 4). Correlation between microarray and RT-PCR results was $0.36\left(\mathrm{R}^{2}=0.74\right), 0.55\left(\mathrm{R}^{2}=0.78\right)$ and $0.77\left(\mathrm{R}^{2}=0.81\right)$ for wild boar infected with $A$. phagocytophilum, B. suis and M. bovis, respectively, and $0.64\left(\mathrm{R}^{2}=0.79\right)$ and $0.70\left(\mathrm{R}^{2}=0.80\right)$ for sheep infected with $A$. phagocytophilum and B. ovis, respectively.

\section{Effect of Anaplasma, Brucella and Mycobacterium infection on wild boar and sheep innate and adaptive immunity}

These results showed that Anaplasma, Brucella and Mycobacterium infection of wild boar and sheep affect the expression of genes involved in host innate and adaptive immunity. However, not surprisingly, the way in which host immune response was affected varied between different host-bacteria interactions. Differences in host immune response between different host-pathogen interactions could be related to host/pathogen-specific factors and/or differences in gene expression between early (acute) and late (chronic) infections. Nevertheless, common to all bacteria-host interactions was the induction of innate immunity through upregulation of pro-inflammatory cytokines such as interleukins IL1B and/or IL6 that are induced in phagocytes after toll-like receptor (TLR) recognition resulting in activation of the complement system and pathogen opsonization for phagocytosis by macrophages and neutrophils [13]. As in previous experiments with cultured human macrophages infected with Gram-positive bacteria, Gram-negative bacteria and M. tuberculosis [2], shared responses included genes encoding receptors and signal transduction molecules affecting the cytokine-receptor interaction, hematopoietic cell lineage and Jak-STAT signaling pathways. However, adaptive immunity was induced through upregulation of genes such as cluster differentiation 4 (CD4) and IL21 only in wild boar and sheep infected with $A$. phagocytophilum.

The results obtained herein showed that the upregulation of host innate immune pro-inflammatory genes and signaling pathways constitute a general antibacterial mechanism in response to pathogenic intracellular bacteria of the genera Anaplasma, Brucella and Mycobacterium, a finding previously suggested in other studies with Brucella spp. [5,8,14], Mycobacterium spp. [2,4,15-20] and A. phagocytophilum $[7,21]$.

Role for the Jak-STAT pathway during Anaplasma, Brucella and Mycobacterium infection of wild boar and sheep

Pathway-focused analysis revealed a role for the Jak-STAT pathway during bacterial intracellular infection, a fact reported before in 
Citation: Galindo RC, de la Fuente J (2012) Transcriptomics Data Integration Reveals Jak-STAT as a Common Pathway Affected by Pathogenic Intracellular Bacteria in Natural Reservoir Hosts. J Proteomics Bioinform 5: 108-115. doi:10.4172/jpb.1000221

\begin{tabular}{|c|c|c|c|}
\hline \multirow{2}{*}{ GenBank accession number ${ }^{1}$} & \multirow{2}{*}{$\begin{array}{l}\text { Gene } \\
\text { symbol }\end{array}$} & \multicolumn{2}{|c|}{ Upstream/downstream primer sequences $\left(5^{\prime}-3^{\prime}\right)$} \\
\hline & & Wild boar (Sus scrofa) & Sheep/Cattle (Ovis aries/Bos taurus) \\
\hline NM_213844.2/ NM_001144097.1 & CRP & $\begin{array}{l}\text { Ss-CRPF: GTGTTGTCACCGGAGGAGAT } \\
\text { Ss-CRPR: CCAGAGACAAGGGGAACGTA }\end{array}$ & $\begin{array}{l}\text { Oa-CRPF: AGCATGTCCCGTACCAAAAG } \\
\text { Oa-CRPR: TTTTTCCTTGACAGTTGCAG }\end{array}$ \\
\hline NM_214155.2/ NM_001009417.1 & CD247 & $\begin{array}{l}\text { Ss-CD247F: TGGGGAAGGACAAGATGAAG } \\
\text { Ss-CD247R: TCTCTCAGGAACAGGGCAGT }\end{array}$ & $\begin{array}{l}\text { Bt-CD247F: TTGTCACTGCCCTGTTTCTG } \\
\text { Bt-CD247R: ACTTCGTGGGGGTTCTTCTT }\end{array}$ \\
\hline $\begin{array}{l}\text { NM_213775.2/ } \\
\text { NM_001009382.1 }\end{array}$ & CD3D & $\begin{array}{l}\text { Ss-CD3DF: TCTCTCAGGAACAGGGCAGT } \\
\text { Ss-CD3DR: AGGGAAGCGAAGAAAGAAGG }\end{array}$ & $\begin{array}{l}\text { Oa-CD3DF: TTGAGGACCCAAGAGGAATG } \\
\text { Oa-CD3DR: GTCTCATGTCCAGCAAAGCA }\end{array}$ \\
\hline $\begin{array}{l}\text { NM_001001908.1/ } \\
\text { NM_001129902.1 }\end{array}$ & CD4 & $\begin{array}{l}\text { Ss-CD4F: GCTGGGGAACCAGAGTATGA } \\
\text { Ss-CDFR: AGAACCCAGCGAGAAACAGA }\end{array}$ & $\begin{array}{l}\text { Oa-CD4F: AAGCTCGAGGTGGAACTGAA } \\
\text { Oa-CD4R: CGTCCAGGTACCACTGTCCT }\end{array}$ \\
\hline NM_213774.1/ NM_001034735.1 & CD74 & $\begin{array}{l}\text { Ss-CD74F: CCTGCTCCTGAAGTCTGACC } \\
\text { Ss-CD74R: GTGTCTCCTCCAGCGAGTTC }\end{array}$ & $\begin{array}{l}\text { Bt-CD74F: TTGAGGGTCCACCAAAAGAC } \\
\text { Bt-CD74R: GCTGATGGAGAGGCAGAGTC }\end{array}$ \\
\hline NM_214269.2/NM_174375.2 & KITLG & $\begin{array}{l}\text { Ss-KITLGF: GATGCCTTCAAGGATTTGGA } \\
\text { Ss-KITLGR: ATGGAATCTGAGGCCTTCCT }\end{array}$ & $\begin{array}{l}\text { Bt-KITLGF: CGTCCACACTCAAGGGATCT } \\
\text { Bt-KITLGR: TTCCACCATCTCGCTTATCC }\end{array}$ \\
\hline NM_214354.1/ NM_001076269.1 & CALCR & $\begin{array}{l}\text { Ss-CALCRF: TGGAATCTCCAATCCAGGAG } \\
\text { Ss-CALCRR: AGCACCAGCGTGTAAGTGTG }\end{array}$ & $\begin{array}{l}\text { Bt-CALCRF: CCCATCCTGAGAGCAACATT } \\
\text { Bt-CALCRR: AACACGCATGAAAATCACCA }\end{array}$ \\
\hline $\begin{array}{l}\text { XM_001924460.1/ } \\
\text { NM_001100293.1 }\end{array}$ & CCR4 & $\begin{array}{l}\text { Ss-CCR4F: TCACAGGAATGGCCTTTTTC } \\
\text { Ss-CCR4R: GACTGCTTGTTGGCTTCCTC }\end{array}$ & $\begin{array}{l}\text { Bt-CCR4F: TGTTCACTGCTGCCTCAATC } \\
\text { Bt-CCR4R: TAAGATGAGCTGGGGGTGTC }\end{array}$ \\
\hline $\begin{array}{l}\text { NM_001009580.1/ } \\
\text { NM_001113174.1 }\end{array}$ & CXCL12 & $\begin{array}{l}\text { Ss-CXCL12F: CAGTGTCCCCAGTGTGTCAG } \\
\text { Ss-CXCL12R: CTCTCAAAGAATCGGCAAGG }\end{array}$ & $\begin{array}{l}\text { Bt-CCXCL12F: GAGATCATGTCTCCGCCTTC } \\
\text { Bt-CCXCL12R: GAAACTGTGCTGTGGCTTCA }\end{array}$ \\
\hline $\begin{array}{l}\text { U61139.1/ } \\
\text { L07939.1 }\end{array}$ & CSF2 & $\begin{array}{l}\text { Ss-CSF2F: TTACCATCCCCTTTGACTGC } \\
\text { Ss-CSF2R: AGTCTGTGCCCCATTACAGC }\end{array}$ & $\begin{array}{l}\text { Oa-CSFF: CGTCCAGGTACCACTGTCCT } \\
\text { Oa-CSFR: GTTGGTCTAGGCAGCTCGTC }\end{array}$ \\
\hline NM_001003924 / NM_001014945 & C1QA & $\begin{array}{l}\text { Ss-C1QAF: CTTCCAGGTGGTGTCCAAGT } \\
\text { Ss-C1QAR: TGGATCCAGACCTTGTCTCC }\end{array}$ & $\begin{array}{l}\text { Bt-C1QAF: GCATCTTCAGTGGCTTCCTC } \\
\text { Bt-C1QAR: ACTTGGTAGGGCAGAGCAGA }\end{array}$ \\
\hline AY349420.1/ NM_001046599 & $\mathrm{C} 1 \mathrm{qB}$ & $\begin{array}{l}\text { Ss-C1QBF: GCGAGTCCGGAGACTACAAG } \\
\text { Ss-C1QBR: ATGAGGTTCACGCACAGGTT }\end{array}$ & $\begin{array}{l}\text { Bt-C1QBF: CTGCGACTACGTCCAGAACA } \\
\text { Bt-C1QBR: GTTGGTGTTGGGGAGAAAGA }\end{array}$ \\
\hline $\begin{array}{l}\text { NM_001001646.1/ } \\
\text { NM_001166616.1 }\end{array}$ & C5 & $\begin{array}{l}\text { Ss-C5F: GCATGTCCCAGACCAAACTT } \\
\text { Ss-C5R: ACGGCTTCTCCAGCTTTGTA }\end{array}$ & $\begin{array}{l}\text { Bt-C5F: TGCTGAGAGAGACGCTGAAA } \\
\text { Bt-C5R: TCAATCCAGGTCGAGGAATC }\end{array}$ \\
\hline NM_214282.1/ NM_001045966.1 & $\mathrm{C} 7$ & $\begin{array}{l}\text { SsC7F: TCAAGTGCCTCCTCTCCTGT } \\
\text { SsC7R: GCTGATGCACTGACCTGAAA }\end{array}$ & $\begin{array}{l}\text { Bt-C7F: GGCGGTCAATTGCTGTTTAT } \\
\text { Bt-CTR: GGTCTGCTTTCTGCATCCTC }\end{array}$ \\
\hline NM_213975.1/ NM_001009786.1 & FTH1 & $\begin{array}{l}\text { Ss-FTH1F: TGCTTCAACAGTGCTTGGAC } \\
\text { Ss-FTH1R: TCTTCAAAGCCACATCATCG }\end{array}$ & $\begin{array}{l}\text { Oa-FTH1F: CGCTACTGGAACTGCACAAA } \\
\text { Oa-FTH1R: CAGGGTGTGCTTGTCAAAGA }\end{array}$ \\
\hline $\begin{array}{l}\text { NM_001004027.1/ } \\
\text { NM_001014912.1 }\end{array}$ & HMOX1 & $\begin{array}{l}\text { Ss-HMOX1F: ATGTGAATGCAACCCTGTGA } \\
\text { Ss-HMOX1R: GTGCTCTTGGTTGGGAAGA }\end{array}$ & $\begin{array}{l}\text { Bt-HMOX1F: ACTCACCCCTTCCTGTTCCT } \\
\text { Bt-HMOX1R: CACAAAGCTGCTCCAACAAA }\end{array}$ \\
\hline NM_001123124.1/NM_174339.3 & HIF1A & $\begin{array}{l}\text { Ss-HIF1AF: TTACAGCAGCCAGATGATCG } \\
\text { Ss-HIF1AR: TGGTCAGCTGTGGTAATCCA }\end{array}$ & $\begin{array}{l}\text { Bt-HIF1AF: TCAGCTATTTGCGTGTGAGG } \\
\text { Bt.HIF1AR: TCGTGGTCACATGGATGAGT }\end{array}$ \\
\hline NM_214055.1/ NM_001009465.2 & IL1B & $\begin{array}{l}\text { Ss-IL1BF: CAGCCATGGCCATAGTACCT } \\
\text { Ss-IL1BR: CCACGATGACAGACACCATC }\end{array}$ & $\begin{array}{l}\text { Oa-IL1BF: CGAACATGTCTTCCGTGATG } \\
\text { Oa-IL1BR: GAAGCTCATGCAGAACACCA }\end{array}$ \\
\hline AY552750.1 / NM_001009734.1 & IL15 & $\begin{array}{l}\text { Ss-IL15F: TTGTCCTGTGTGTTCGGTGT } \\
\text { Ss-IL15R: GCAAACCTTTTGAGTGAGC }\end{array}$ & $\begin{array}{l}\text { Oa-IL15F: TTTGGGCTGTATCAGTGCAG } \\
\text { Oa-IL15R: AATAACGCGTAGCTCGAGGA }\end{array}$ \\
\hline NM_214415.1/NM_198832.1 & IL21 & $\begin{array}{l}\text { Ss-IL21F: CGGGGAACATGGAGAAAATA } \\
\text { Ss-IL21R: CAGCAATTCAGGGTCCAAGT }\end{array}$ & $\begin{array}{l}\text { Bt-IL21F: CGGGGAACATGGAGAGAATA } \\
\text { Bt-IL21R: GGCAGAAATTCAGGATCCAA }\end{array}$ \\
\hline $\begin{array}{l}\text { BU946820.1/ } \\
\text { NM_001195219.1 }\end{array}$ & IL25 & $\begin{array}{l}\text { Ss-IL25F: CTCACCTGCGTGTCACCTT } \\
\text { Ss-IL25R: AATATGGCATGGCCTACTCG }\end{array}$ & $\begin{array}{l}\text { Oa-IL25F: GCCCCCTGGAGATATGAGTT } \\
\text { Oa-IL25R: AGAAAACGGTCTGGTTGTGG }\end{array}$ \\
\hline NM_214340.1/ NM_001075142.1 & IL4R & $\begin{array}{l}\text { Ss-IL4RF: CCCATCTGCCTATCCGACTA } \\
\text { Ss-IL4RR: TGACAATGCTCTCCATCAGC }\end{array}$ & $\begin{array}{l}\text { Bt-IL4F: CTGAGCCCAGAGTCAAGTCC } \\
\text { Bt-IL4R: CAGCTGTGGGTCTGAGTCAA }\end{array}$ \\
\hline NM_214205.1/ NM_001009783.1 & IL5 & $\begin{array}{l}\text { Ss-IL5F: TGGCAGAGACCTTGACACTG } \\
\text { Ss-IL5R: CCCTCGTGCAGTTTGATTCT }\end{array}$ & $\begin{array}{l}\text { Oa-IL5F: AAAGGCAAACGCTGAACATT } \\
\text { Oa-IL5R: CAGAGTTTGATGCGTGGAGA }\end{array}$ \\
\hline M80258.1/NM_001009392.1 & IL6 & $\begin{array}{l}\text { Ss-IL6F: CACCAGGAACGAAAGAGAGC } \\
\text { Ss-IL6R: GTTTTGTCCGGAGAGGTGAA }\end{array}$ & $\begin{array}{l}\text { Oa-IL6F: TGGAGGAAAAAGATGGATGC } \\
\text { Oa-IL6R: TGCATCTTCTCCAGCATGTC }\end{array}$ \\
\hline NM_001166043.1/El184569.1 & IL9 & $\begin{array}{l}\text { Ss-IL9F: TATGTCTGCCCATTCCTTCC } \\
\text { Ss-IL9R: CATGGCTGTTCACAGGAAAA }\end{array}$ & $\begin{array}{l}\text { Oa-IL9F: CACCACCACACTTTTGCATC } \\
\text { Oa-IL9R: ACCCACCCAGAGAGGAATCT }\end{array}$ \\
\hline
\end{tabular}


Citation: Galindo RC, de la Fuente J (2012) Transcriptomics Data Integration Reveals Jak-STAT as a Common Pathway Affected by Pathogenic Intracellular Bacteria in Natural Reservoir Hosts. J Proteomics Bioinform 5: 108-115. doi:10.4172/jpb.1000221

\begin{tabular}{|c|c|c|c|}
\hline NM_001077213.2/NM_001078655.1 I & MIF & $\begin{array}{l}\text { Ss-MIFF: GAACCGTTCCTACAGCAAGC } \\
\text { SS-MIFR: CCGAGAGCAAAGGAGTCTTG }\end{array}$ & $\begin{array}{l}\text { Oa-MIFF: CTCCTCTCCGAGCTCACG } \\
\text { Oa-MIFR: TGTAGATCCTGTCCGGGCTA }\end{array}$ \\
\hline $\begin{array}{l}\text { NM_001009578.1/ } \\
\text { NM_001046477.1 }\end{array}$ & MSN & $\begin{array}{l}\text { Ss-MSNF: TGACCCCACACACTCCTACA } \\
\text { Ss-MSNR: CCATAGTGGGCCATCTGTCT }\end{array}$ & $\begin{array}{l}\text { Bt-MSNF: AAGGAGAGTGAGGCTGTGGA } \\
\text { Bt-MSNR: CCCATTCTCATCCTGCTCAT }\end{array}$ \\
\hline NM_214379.1/NM_001100921.1 & PPARG & $\begin{array}{l}\text { Ss-PPARGF: GCCCTTCACCACTGTTGATT } \\
\text { Ss-PPARGR: GAGTTGGAAGGCTCTTCGTG }\end{array}$ & $\begin{array}{l}\text { Oa-PPARGF: CCCTGGCAAAGCATTTGTAT } \\
\text { Oa-PPARGR: ACTGACACCCCTGGAAGATG }\end{array}$ \\
\hline AF527990.2/ ES414801.1 & PSME1 & $\begin{array}{l}\text { Ss-PSME1F: AAGAAGGGGGAAGATGAGGA } \\
\text { Ss-PSME1R: CTTCTCCTGGACAGCCACTC }\end{array}$ & $\begin{array}{l}\text { Oa-PSMEF: AAGCCAAGGTGGATGTGTTC } \\
\text { Oa-PSMER: AGGCACTGGGATGTCCAAT }\end{array}$ \\
\hline AF139837.1/ XM_002693929.1 & RGS1 & $\begin{array}{l}\text { Ss-RGS1F: GAGTCCGATCTTTTGCATCG } \\
\text { Ss-RGS1R: TGATTTTCTGGGCTTCATCA }\end{array}$ & $\begin{array}{l}\text { Bt-RGS1F: GTGGTCTGAATCCCTGGAAA } \\
\text { Bt-RGS1R: GATTCTCGAGTGCGGAAGTC }\end{array}$ \\
\hline NM_001012299.1/ NM_174176.2 & SCG2 & $\begin{array}{l}\text { Ss-SCG2F: CATGCGTTTCCCTCCTATGT } \\
\text { Ss-SCG2R: TCTCACGCTTCTGGTTGTTG }\end{array}$ & $\begin{array}{l}\text { Bt-SCG2F: ACTGGAGAGAAGCCAGTGGA } \\
\text { Bt-SCG2R: TATGGAGGCTTTGGATTTGC }\end{array}$ \\
\hline AB258452.1/ GQ175957.1 & TLR8 & $\begin{array}{l}\text { Ss-TLR8F: TGTCATTGCAGAGTGCAACA } \\
\text { Ss-TLR8R: GAGAAACGCCCCATCTGTAA }\end{array}$ & $\begin{array}{l}\text { Oa-TLR8F: CCTTGCAGAGGCTAATGGAG } \\
\text { Oa-TLR8R: CTCTGCCAAAACAAGCCTTC }\end{array}$ \\
\hline L43124.1/NM_174484.1 & VCAM1 & $\begin{array}{l}\text { Ss-VCAMF: ATCCAAGCTGCTCCAAAAGA } \\
\text { Ss-VCAMR: GGCCCTGTGGATGGTATATG }\end{array}$ & $\begin{array}{l}\text { Bt-VCAMF: GAACCGACAGCTCCTTTCTG } \\
\text { BT-VCAMR: TCCCTGACATCACAGGTCAA }\end{array}$ \\
\hline $\begin{array}{l}\text { NM_001031797.1/ } \\
\text { NM_001123003.1 }\end{array}$ & FADD & $\begin{array}{l}\text { Ss-FADDF: AGTATCCCCGAAACCTGACC } \\
\text { Ss-FADDR: CAGGAAATGAGGGACACAGG }\end{array}$ & $\begin{array}{l}\text { Oa-FADDF: TGCAGATATTGCTTGGCTTG } \\
\text { Oa-FADDR: CAGCATTCATCTCCCCAACT }\end{array}$ \\
\hline NM_001014971.1 & COL5A1 & $\begin{array}{l}\text { Ss-COL5F: GGAGATCGAGCAGATGAAGC } \\
\text { Ss-COL5R: GCCCCTTCGGACTTCTTATC }\end{array}$ & Sequence not available for $O$. aries or $B$. taurus \\
\hline U83916.1/ NM_001164714.1 & CTGF & $\begin{array}{l}\text { Ss-CTGFF: CATGGCCTAAAGCCAGAGAG } \\
\text { Ss-CTGFR: TGGCACACGATTTTGAATGT }\end{array}$ & $\begin{array}{l}\text { Oa-CTGFF: CCTGGTCCAGACCACAGAGT } \\
\text { Oa-CTGFR: GCAGCCAGAGAGCTCAAACT }\end{array}$ \\
\hline NM_001129953.1/ U47636.1 & DMP1 & $\begin{array}{l}\text { Ss-DMP1F: CACTGAATCCGAAGAGCACA } \\
\text { Ss-DMP1R: CCTGGATTGTGTGGTGTCAG }\end{array}$ & $\begin{array}{l}\text { Oa-DMP1F: AGCCCAGAGTCCACTGAAGA } \\
\text { Oa-DMP1R: GTTTGTTGTGGTACGCATCG }\end{array}$ \\
\hline AJ577089.1/ NM_001009769.1 & FGF2 & $\begin{array}{l}\text { Ss-FGF2F: AGCGACCCTCACATCAAACT } \\
\text { Ss-FGG2R: TCGTTTCAGTGCCACATACC }\end{array}$ & $\begin{array}{l}\text { Oa-FGF2F: GTGCAAACCGTTACCTTGCT } \\
\text { Oa-FGF2R: ACTGCCCAGTTCGTTTCAGT }\end{array}$ \\
\hline AF052657.1/ NM_001009235.1 & FGF7 & $\begin{array}{l}\text { Ss-FGF7F: TTTGCTGAACCCAATTCCTC } \\
\text { Ss-FGF7R: CAGGAACCCCCTTTTGATTT }\end{array}$ & $\begin{array}{l}\text { Oa-FGF7F: ATGAACACCCGGAGCACTAT } \\
\text { Oa-FGF7R: GGGCTGGAACAGTTCACATT }\end{array}$ \\
\hline NM_001103212.1/ NM_176669.3 & STC1 & $\begin{array}{l}\text { Ss-STC1F: GCTCTACTTTCCAGCGGATG } \\
\text { Ss-STC1R: TCTTCGTCACATTCCAGCAG }\end{array}$ & $\begin{array}{l}\text { Bt-STC1F: AGCTGAACGTGTGCAGTGTC } \\
\text { Bt-STC1R: CGTCTGCAGGATGTGAAAGA }\end{array}$ \\
\hline NM_214198.1/ AY656798.1 & TGFB3 & $\begin{array}{l}\text { Ss-TGFB3F: GATGAGCACATAGCCAAGCA } \\
\text { Ss-TGFB3R: AGGTGTGACACGGACAATGA }\end{array}$ & $\begin{array}{l}\text { Oa-TGFB3F: AGCGGTATATCGATGGCAAG } \\
\text { Oa-TGFB3R: ATTGGGCTGAAAGGTGTGAC }\end{array}$ \\
\hline NM_001114670.1/NM_001191344.1 & TKY2 & $\begin{array}{l}\text { Ss-TKY2F: ACTGCTATGACCCGACCAAC } \\
\text { Ss-TKY2R: TGACTTCTCGCCTTGGTCTT }\end{array}$ & $\begin{array}{l}\text { Oa-FLT4F: AGCTAGCCACTCCTGCCATA } \\
\text { Oa-FLT4R: TCTGTGTCAGCATCCGTCTC }\end{array}$ \\
\hline NM_214292.1/ AY029232.1 & EPOR & $\begin{array}{l}\text { Ss-EPORF: CTACCAGCTTGAGGGTGAGC } \\
\text { Ss-EPORR: CCACTTCGTTGATGTGGATG }\end{array}$ & $\begin{array}{l}\text { Oa-EPORF: GTTGGTCTAGGCAGCTCGTC } \\
\text { Oa-EPORR: TACTCAAAGCTGGCAGCAGA }\end{array}$ \\
\hline DQ450679.1/XM_002692067.1 & IL15RA & $\begin{array}{l}\text { Ss-IL15F: TTGTCCTGTGTGTTCGGTGT } \\
\text { Ss-IL15R: GCAAAGCCTTTTGAGTGAGC }\end{array}$ & $\begin{array}{l}\text { Bt-IL15RAF: AGGCTCCGGAACACACATAC } \\
\text { Bt-IL15RAR: CACACTCTCCATGCTCTCCA }\end{array}$ \\
\hline AY008846/AJ865374.1 & Cyclophilin & $\begin{array}{l}\text { SsCYCLOPHILINL: AGCACTGGGGAGAAAGGATT } \\
\text { SsCYCLOPHILINR: CTTGGCAGTGCAAATGAAAA }\end{array}$ & $\begin{array}{l}\text { Oa-CyclophBF: CTTGGCTAGACGGCAAACAT } \\
\text { Oa-CyclophBR: GCTTCTCCACCTCGATCTTG }\end{array}$ \\
\hline DQ845171/ U39357 & Beta-actin & $\begin{array}{l}\text { SusBetActin-L: GACATCCGCAAGGACCTCTA } \\
\text { SusBetActin-R: ACACGGAGTACTTGCGCTCT }\end{array}$ & $\begin{array}{l}\text { ACTOVI5: CTCTTCCAGCCTTCCTTCCT } \\
\text { ACTOVI3:GGGCAGTGATCTCTTTCTGC }\end{array}$ \\
\hline
\end{tabular}

${ }^{1}$ GenBank accession numbers are shown for wild boar/sheep-cattle sequences.

Table 2: Primer sets used for analysis of differential gene expression by real-time RT-PCR.

Mycobacterium-infected cells [22-24] but not during Brucella spp. and A. phagocytophilum infection. This result highlighted the importance of integrating data from different trascriptomics experiments to discover common host-cell mechanisms affected by pathogenic intracellular bacteria.

In mammals, the Jak-STAT pathway is the principal signaling mechanism for a wide array of cytokines and growth factors such as CSF2, IL15, IL21, IL4R, IL5, IL6, IL9, TKY2, EPOR, IL15RA shown here to be differentially expressed in infected animals [25]. Jak activation stimulates cell proliferation, differentiation, cell migration and apoptosis resulting in hematopoiesis and immune development among other processes [25]. Predictably, downregulation of the JakSTAT pathway activity affect these processes but failure to properly regulate Jak signaling cause inflammation, erythrocytosis and leukemia among other diseases [25]. Herein, a clear activation of the Jak-STAT pathway was observed in A. phagocytophilum-infected wild boar and sheep when compared to uninfected controls (Table 4). For Brucella spp., infection resulted in the upregulation of some ligands and the 
Citation: Galindo RC, de la Fuente J (2012) Transcriptomics Data Integration Reveals Jak-STAT as a Common Pathway Affected by Pathogenic Intracellular Bacteria in Natural Reservoir Hosts. J Proteomics Bioinform 5: 108-115. doi:10.4172/jpb.1000221

\begin{tabular}{|c|c|c|c|c|}
\hline Term & Count $^{1}$ & $P$ value ${ }^{2}$ & Fold change $^{3}$ & Benjamini $^{4}$ \\
\hline \multicolumn{5}{|c|}{ Biological process $\left(\mathrm{ES}^{5}=9.68\right)$} \\
\hline Regulation of immune system & 21 & $1.5 \mathrm{E}-14$ & 9.7 & 2.2E-11 \\
\hline Immune system & 30 & $2.1 \mathrm{E}-14$ & 5.4 & $1.6 \mathrm{E}-11$ \\
\hline \multicolumn{5}{|c|}{ Molecular function $(E S=10.27)$} \\
\hline Receptor binding & 28 & $6.4 \mathrm{E}-15$ & 6.2 & $1.8 \mathrm{E}-12$ \\
\hline Cytokine activity & 12 & $3.4 \mathrm{E}-09$ & 12.0 & 4.7E-07 \\
\hline Growth factor activity & 11 & 7.3E-09 & 13.0 & $5.1 \mathrm{E}-07$ \\
\hline \multicolumn{5}{|c|}{ Pathway $(E S=3.70)$} \\
\hline Cytokine-cytokine receptor interaction & 16 & $6.0 \mathrm{E}-08$ & 5.5 & $3.8 \mathrm{E}-06$ \\
\hline Hematopoietic cell lineage & 9 & 3.1E-06 & 9.5 & $9.8 \mathrm{E}-05$ \\
\hline Jak-STAT signaling pathway & 10 & $3.4 \mathrm{E}-05$ & 5.9 & 4.4E-04 \\
\hline
\end{tabular}

${ }^{1}$ Indicates the number of genes involved in individual GO terms. ${ }^{2}$ Defines the significance of a GO term enrichment with a modified Fisher's exact test (EASE score), denoting if the term is over or under represented (if $\mathrm{P} \leq 0.05$, then terms are overrepresented). ${ }^{3}$ Statistical threshold for $\mathrm{GO}$ term selection (FC $>2$ ). ${ }^{4} \mathrm{To}$ globally correct enrichment P-values to control family-wide false discovery rate at Benjamini $\leq 0.0004$. ${ }^{5}$ Enrichment score (ES) was used to rank overall importance (enrichment) of GO terms.

Table 3: Common host-cell biological processes, molecular functions and pathways affected by pathogenic intracellular bacteria.

\begin{tabular}{|c|c|c|c|c|c|c|}
\hline \multirow{2}{*}{ Gene symbol } & \multirow{2}{*}{ Gene description } & \multicolumn{5}{|c|}{ Host-bacteria interaction } \\
\hline & & WB-A & WB-B & WB-M & S-A & S-B \\
\hline CRP & C-reactive protein, pentraxin-related & $1.6(\mathrm{~ns})$ & ns (ns) & $-2.0(-3.3 \pm 0.01)$ & ns (ns) & ns (ns) \\
\hline CD247 & CD247 molecule & $1.6(\mathrm{~ns})$ & $-2.2(\mathrm{~ns})$ & ns (ns) & ns (ns) & ns (ns) \\
\hline CD3D & CD3d molecule, delta (CD3-TCR complex) & ns (ns) & ns (ns) & $2.1(\mathrm{~ns})$ & $-2.2(\mathrm{~ns})$ & ns (ns) \\
\hline CD4 & CD4 molecule & $1.4(\mathrm{~ns})$ & ns (ns) & ns (ns) & $1.3(\mathrm{~ns})$ & ns (ns) \\
\hline CD74 & CD74 molecule, major histocompatibility complex. & ns (ns) & $-4.1(\mathrm{~ns})$ & $-3.3(-5.3 \pm 0.2)$ & ns (ns) & ns (ns) \\
\hline KITLG & KIT ligand & $3.2(\mathrm{~ns})$ & ns (ns) & ns (ns) & $-1.2(\mathrm{~ns})$ & ns (ns) \\
\hline CALCR & Calcitonin receptor & $5.3(\mathrm{~ns})$ & 3.7 (ns) & ns (ns) & ns (ns) & ns (ns) \\
\hline CCR4 & Chemokine (C-C motif) receptor 4 & ns $(1.9 \pm 0.02)$ & ns (ns) & $-2.3(-8.1 \pm 0.05)$ & $-1.4(\mathrm{~ns})$ & $3.0(\mathrm{~ns})$ \\
\hline CXCL12 & $\begin{array}{l}\text { Chemokine (C-X-C motif) ligand } 12 \text { (stromal cell- } \\
\text { derived factor 1) }\end{array}$ & $1.4(\mathrm{~ns})$ & $-4.8(\mathrm{~ns})$ & $-9.8(-2.5 \pm 0.02)$ & $-2.7(-4.5 \pm 2 \mathrm{E}-3)$ & ns $(-11.1 \pm 8 E-6)$ \\
\hline CSF2 & Colony stimulating factor 2 (granulocyte-macrophage) & $1.4(\mathrm{~ns})$ & ns (ns) & ns (ns) & $1.6(6.1 \pm 4 \mathrm{E}-4)$ & ns $(-4.2 \pm 4.5-6)$ \\
\hline C1QA & Complement component 1 , q subcomponent, A chain & $1.3(\mathrm{~ns})$ & $-2.8(\mathrm{~ns})$ & ns $(-3.7 \pm 0.2)$ & ns (ns) & $\mathrm{ns}(\mathrm{ns})$ \\
\hline C1qB & Complement component 1 , q subcomponent, $B$ chain & $1.3(\mathrm{~ns})$ & $-3.9(\mathrm{~ns})$ & $-9.0(\mathrm{~ns})$ & ns (ns) & ns (ns) \\
\hline C5 & Complement component 5 & 1.5 (ns) & ns ns & ns ns & $1.3(8.0 \pm 4 \mathrm{E}-4)$ & ns $(-10.0 \pm 2 E-6)$ \\
\hline $\mathrm{C} 7$ & Complement component 7 & 2.5 (ns) & $\mathrm{ns}(\mathrm{ns})$ & $\mathrm{ns}(\mathrm{ns})$ & $1.3(\mathrm{~ns})$ & $\mathrm{ns}(\mathrm{ns})$ \\
\hline FTH1 & Ferritin, heavy polypeptide 1 & ns (ns) & $-3.6(\mathrm{~ns})$ & $-4.1(-2.5 \pm 0.7)$ & ns (ns) & ns (ns) \\
\hline HMOX1 & Heme oxygenase (decycling) 1 & ns (ns) & $-2.5(\mathrm{~ns})$ & $-1.7(-2.5 \pm 0.1)$ & ns (ns) & ns (ns) \\
\hline HIF1A & $\begin{array}{l}\text { Hypoxia inducible factor } 1 \text {, alpha subunit (basic helix- } \\
\text { loop-helix transcription factor) }\end{array}$ & $1.3(\mathrm{~ns})$ & $-2.0(-3.0 \pm 0.02)$ & ns (ns) & ns (ns) & ns (ns) \\
\hline IL1B & Interleukin 1, Beta & ns (ns) & 2.9 (ns) & ns (ns) & $1.3(2.3 \pm 3 E-4)$ & $2.1(1.4 \pm 5 E-5)$ \\
\hline IL15 & Interleukin 15 & ns (ns) & ns (ns) & ns (ns) & $1.2(\mathrm{~ns})$ & 2.7 (ns) \\
\hline IL21 & Interleukin 21 & $1.3(\mathrm{~ns})$ & ns (ns) & ns (ns) & $1.2(\mathrm{~ns})$ & ns (ns) \\
\hline IL25 & Interleukin 25 & ns (ns) & $\mathrm{ns}(\mathrm{ns})$ & ns (ns) & $1.3(\mathrm{~ns})$ & $1.6(\mathrm{~ns})$ \\
\hline IL4R & Interleukin 4 receptor & $1.2(\mathrm{~ns})$ & ns (ns) & ns (ns) & $-2.0(\mathrm{~ns})$ & ns (ns) \\
\hline IL5 & Interleukin 5 (colony-stimulating factor, eosinophil) & $1.3(\mathrm{~ns})$ & ns $(10.8 \pm 0.5)$ & ns $(-3.1 \pm 3 E-4)$ & ns (ns) & $2.6(\mathrm{~ns})$ \\
\hline IL6 & Interleukin 6 (interferon, beta 2) & $2.0(\mathrm{~ns})$ & ns (ns) & ns (ns) & $1.2(\mathrm{~ns})$ & ns $(1.1 \pm 4 \mathrm{E}-6)$ \\
\hline IL9 & Interleukin 9 & ns (ns) & ns (ns) & ns (ns) & $1.3(\mathrm{~ns})$ & $1.3(\mathrm{~ns})$ \\
\hline MIF & $\begin{array}{l}\text { Macrophage migration inhibitory factor (glycosylation- } \\
\text { inhibiting factor) }\end{array}$ & ns (ns) & $\mathrm{ns}(\mathrm{ns})$ & ns (ns) & $-2.0(\mathrm{~ns})$ & $10.4(\mathrm{~ns})$ \\
\hline MSN & Moesin & ns (ns) & $-2.6(n s)$ & $-3.4(\mathrm{~ns})$ & ns (ns) & $\mathrm{ns}(\mathrm{ns})$ \\
\hline PPARG & Peroxisome proliferator-activated receptor gamma & $1.2(\mathrm{~ns})$ & $-3.5(\mathrm{~ns})$ & ns (ns) & ns (ns) & ns (ns) \\
\hline
\end{tabular}


Citation: Galindo RC, de la Fuente J (2012) Transcriptomics Data Integration Reveals Jak-STAT as a Common Pathway Affected by Pathogenic Intracellular Bacteria in Natural Reservoir Hosts. J Proteomics Bioinform 5: 108-115. doi:10.4172/jpb.1000221

\begin{tabular}{|c|c|c|c|c|c|c|}
\hline PSME1 & Proteasome (prosome, macropain) activator subunit 1 & ns $(3.4 \pm 2)$ & $-3.1(n s)$ & $-2.4(-3.5 \pm 2.8)$ & ns (ns) & ns (ns) \\
\hline RGS1 & Regulator of G-protein signaling 1 & ns (ns) & $-2.0(n s)$ & $-1.9(n s)$ & ns (ns) & ns (ns) \\
\hline SCG2 & Secretogranin II (chromogranin C) & $1.6 \pm(n s)$ & $2.1(\mathrm{~ns})$ & ns (ns) & ns (ns) & ns (ns) \\
\hline TLR8 & Toll-like receptor 8 & ns (ns) & $-2.4(\mathrm{~ns})$ & ns $(-2.72 \pm 0.2)$ & $1.2(\mathrm{~ns})$ & ns (ns) \\
\hline VCAM1 & Vascular cell adhesion molecule 1 & $1.6(-3.2 \pm 0.02)$ & $-4.8(n s)$ & $\mathrm{ns}(-2.5 \pm 0.2)$ & ns (ns) & ns (ns) \\
\hline FADD & Fas (TNFRSF6)-associated via death domain & $1.2(\mathrm{~ns})$ & ns (ns) & $-10.8(-2.8 \pm 0.6)$ & ns (ns) & ns (ns) \\
\hline COL5A1 & Collagen, type V, alpha 1 & $1.4(\mathrm{~ns})$ & $-2.5(\mathrm{~ns})$ & ns (ns) & ns (ns) & ns (ns) \\
\hline CTGF & Connective tissue growth factor & $1.3(1.3 \pm 0.3)$ & $-2.8(\mathrm{~ns})$ & ns (ns) & ns (ns) & ns (ns) \\
\hline DMP1 & Dentin matrix acidic phosphoprotein 1 & $1.2(\mathrm{~ns})$ & $1.9(3.6 \pm 0.05)$ & ns (ns) & ns (ns) & ns (ns) \\
\hline FGF2 & Fibroblast growth factor 2 (basic) & $1.6(\mathrm{~ns})$ & ns (ns) & ns $(-3.1 \pm 2 E-3)$ & $1.3(\mathrm{~ns})$ & $1.6(\mathrm{~ns})$ \\
\hline FGF7 & $\begin{array}{l}\text { Hypothetical fibroblast growth factor } 7 \text { (keratinocyte } \\
\text { growth factor) }\end{array}$ & $1.4(\mathrm{~ns})$ & $\mathrm{ns}(\mathrm{ns})$ & ns $(-2.6 \pm 0.1)$ & $1.2(1.9 \pm 7 E-5)$ & ns $(1.9 \pm 2 E-5)$ \\
\hline STC1 & Stanniocalcin 1 & $1.4(\mathrm{~ns})$ & $2.2(12.1 \pm 0.01)$ & ns (ns) & ns (ns) & ns (ns) \\
\hline TGFB3 & Transforming growth factor, beta 3 & $1.3(7.3 \pm 3 E-4)$ & ns (ns) & ns (ns) & ns (ns) & $3.2(1.8 \pm 4 \mathrm{E}-6)$ \\
\hline TKY2 & Tyrosine kinase 2 & ns (ns) & $-1.9(n s)$ & $-2(-2.4 \pm 0.03)$ & ns (ns) & ns (ns) \\
\hline EPOR & Ethropoietin receptor & $1.4(\mathrm{~ns})$ & ns (ns) & ns (ns) & $1.8(\mathrm{~ns})$ & ns (ns) \\
\hline IL15RA & Interleukin 15 receptor, alpha & ns (ns) & ns (ns) & ns (ns) & $1.4(\mathrm{~ns})$ & $-1.6(n s)$ \\
\hline
\end{tabular}

Data shows significant $(P<0.05)$ fold change in differential gene expression (positive and negative values for upregulated and downregulated genes in infected animals, respectively) obtained in the microarray analyses and by real-time RT-PCR (shown in parenthesis; average \pm SD). Abbreviations: ns, not significant differences in gene expression levels between infected and uninfected animals; WB-A, wild boar infected with $A$. phagocytophilum; WB-B, wild boar infected with $B$. suis; WB-M, wild boar infected with $M$. bovis; S-A, sheep infected with $A$. phagocytophilum; S-B, sheep infected with $B$. ovis

Table 4: Differential expression of genes in common host-cell biological processes, molecular functions and pathways affected by pathogenic intracellular bacteria.

downregulation of others that may result in a balanced regulation of the Jak-STAT signaling to prevent negative effects associated with improper regulation of this pathway (Table 4). As previously reported [22-24], M. bovis infection of wild boar clearly produced a downregulation of some of the Jak-STAT effectors such as IL5 and TKY2 (Table 4).

\section{Conclusions}

These results suggested that mycobacteria and brucellae induce host innate immune responses while manipulating adaptive immunity through Jak-STAT pathway and other mechanisms to circumvent hostcell defenses and establish infection. In contrast, A. phagocytophilum infection induces both innate and adaptive immunity, those suggesting that this pathogen uses other mechanisms to circumvent host-cell defenses. These mechanisms may include dowregulation of other adaptive immune genes such as IL2 and IL4 [7,26] and delaying the apoptotic death of neutrophils $[7,21,27,28]$ through activation of the Jak-STAT pathway among other mechanisms.

These results improved our understanding of host-pathogen interactions by characterizing common host-cell mechanisms affected by pathogenic intracellular bacteria of the genera Anaplasma, Brucella and Mycobacterium in natural reservoir hosts and provided insights into mechanisms of pathogenesis that could be used as targets for therapeutic intervention and vaccine development. In fact, some of the cytokine-receptor interactions described here such as those involving IL4 and IL6 have already been used to characterize the immune response to parenteral and oral Bacillus Calmette-Guérin (BCG) vaccination to prevent $M$. bovis infection in wild boar [6,29] and the protective response to the $B$. melitensis $\operatorname{Rev} 1$ vaccine in sheep for the control of B. ovis [9], respectively.

\section{Acknowledgements}

We thank members of our laboratories for fruitful discussions and technical assistance. This research was supported by the Grupo Santander and Fundación
Marcelino Botín, Spain (project Control of Tuberculosis in Wildlife) and the EU FP7, ANTIGONE project number 278976. R.C. Galindo was funded by Ministerio de Ciencia y Educación (MEC), Spain.

\section{References}

1. Bhavsar AP, Guttman JA, Finlay BB (2007) Manipulation of host-cell pathways by bacterial pathogens. Nature 449: 827-834.

2. Nau GJ, Richmond JF, Schlesinger A, Jennings EG, Lander ES, et al. (2002) Human macrophage activation programs induced by bacterial pathogens. Proc Natl Acad Sci USA 5: 1503-1508.

3. Naranjo V, Höfle U, Vicente J, Martín MP, Ruiz-Fons F, et al. (2006) Genes differentially expressed in oropharyngeal tonsils and mandibular lymph nodes of tuberculous and non-tuberculous European wild boars naturally exposed to Mycobacterium bovis. FEMS Immunol Med Microbiol 46: 298-312.

4. Galindo RC, Ayoubi P, Naranjo V, Gortazar C, Kocan KM, et al. (2009) Gene expression profiles of European wild boar naturally infected with Mycobacterium bovis. Vet Immunol Immunopathol 129: 119-125.

5. Galindo RC, Muñoz PM, de Miguel MJ, Marin CM, Labairu J, et al. (2010) Gene expression changes in spleens of the wildlife reservoir species, Eurasian wild boar (Sus scrofa), naturally infected with Brucella suis biovar 2. J Gen Genomics 37: 725-736.

6. Lastra JM, Galindo RC, Gortázar C, Ruiz-Fons F, de la Fuente J, et al. (2009) Expression of immunoregulatory genes in peripheral blood mononuclear cells of European wild boar immunized with BCG. Vet Microbiol 134: 334-339.

7. Galindo RC, Ayoubi P, García-Pérez AL, Naranjo V, Kocan KM, et al. (2008) Differential expression of inflammatory and immune response genes in sheep infected with Anaplasma phagocytophilum. Vet Immunol Immunopathol 126 27-34.

8. Galindo RC, Muñoz PM, de Miguel MJ, Marin CM, Blasco JM, et al. (2009) Differential expression of inflammatory and immune response genes in rams experimentally infected with a rough virulent strain of Brucella ovis. Ve Immunol Immunopathol 127: 295-303.

9. Galindo RC, Muñoz PM, de Miguel MJ, Marin CM, Blasco JM, et al. (2009) Characterization of possible correlates of protective response against Brucella ovis infection in rams immunized with the $B$. melitensis Rev 1 vaccine. Vaccine 27: $3039-3044$

10. Dennis G Jr, Sherman BT, Hosack DA, Yang J, Gao W, et al. (2003) DAVID: 
Citation: Galindo RC, de la Fuente J (2012) Transcriptomics Data Integration Reveals Jak-STAT as a Common Pathway Affected by Pathogenic Intracellular Bacteria in Natural Reservoir Hosts. J Proteomics Bioinform 5: 108-115. doi:10.4172/jpb.1000221

Database for Annotation, Visualization, and Integrated Discovery. Genome Biol 4: P3.

11. Huang DW, Sherman BT, Lempicki RA (2009) Systematic and integrative analysis of large gene lists using DAVID Bioinformatics Resources. Nat Protoc 4: 44-57.

12. Livak KJ, Schmittgen TD (2001) Analysis of relative gene expression data using real-time quantitative PCR and the 2(-Delta Delta $\mathrm{C}(\mathrm{T})$ ) Method. Methods 25: 402-408

13. Medzhitov R (2007) Recognition of microorganisms and activation of the immune response. Nature 449: 819-826

14. Eskra L, Mathison A, Splitter G (2003) Microarray analysis of mRNA levels from RAW264.7 macrophages infected with Brucella abortus. Infect Immun 71: 1125-1133.

15. Goto Y, Nakamura RM, Takahashi H, Tokunaga T (1984) Genetic control of resistance to Mycobacterium intracellular infection in mice. Infect Immun 46: $135-140$

16. Ragno S, Romano M, Howell S, Pappin DJ, Jenner PJ, et al. (2001) Changes in gene expression in macrophages infected with Mycobacterium tuberculosis: a combined transcriptomic and proteomic approach. Immunology 104: 99-108.

17. Keller C, Lauber J, Blumenthal A, Buer J, Ehlers S (2004) Resistance and susceptibility to tuberculosis analyzed at the transcriptome level: lessons from mouse macrophages. Tuberculosis (Edinb) 84: 144-158.

18. Koul A, Herget T, Klebl B, Ullrich A (2004) Interplay between mycobacteria and host signalling pathways. Nat Rev Microbiol 2: 189-202.

19. Naranjo V, Villar M, Martín-Hernando MP, Vidal D, Höfle U, et al. (2007) Proteomic and transcriptomic analyses of differential stress/inflammatory responses in mandibular lymph nodes and oropharyngeal tonsils of European wild boars naturally infected with Mycobacterium bovis. Proteomics 7: 220-231.

20. Fernández de Mera IG, Pérez de la Lastra JM, Ayoubi $P$, Naranjo V, Kocan $\mathrm{KM}$, et al. (2008) Differential expression of inflammatory and immune response genes in mesenteric lymph nodes of Iberian red deer (Cervus elaphus hispanicus) naturally infected with Mycobacterium bovis. Dev Comp Immuno 32: 85-91.

21. Carlyon JA, Fikrig E (2003) Invasion and survival strategies of Anaplasma phagocytophilum. Cell Microbiol 5: 743-754.

22. Jacobsen M, Repsilber D, Kleinsteuber K, Gutschmidt A, Schommer-Leitner $\mathrm{S}$, et al. (2011) Suppressor of cytokine signaling-3 is affected in T-cells from tuberculosisTB patients. Clin Microbiol Infect 17: 1323-1331.

23. Imai K, Kurita-Ochiai T, Ochiai K (2003) Mycobacterium bovis bacillus Calmette-Guérin infection promotes SOCS induction and inhibits IFN-gammastimulated JAK/STAT signaling in J774 macrophages. FEMS Immunol Med Microbiol 39: 173-180.

24. Manca C, Tsenova L, Freeman S, Barczak AK, Tovey M, et al. (2005) Hypervirulent $M$. tuberculosis W/Beijing strains upregulate type I IFNs and increase expression of negative regulators of the Jak-Stat pathway. $J$ Interferon Cytokine Res 25: 694-701.

25. Rawlings JS, Rosler KM, Harrison DA (2004) The JAK/STAT signaling pathway. J Cell Sci 117: 1281-1283.

26. Kim HY, Mott J, Zhi N, Tajima T, Rikihisa Y (2002) Cytokine gene expression by peripheral blood leukocytes in horses experimentally infected with Anaplasma phagocytophila. Clin Diagn Lab Immunol 9: 1079-1084.

27. Borjesson DL, Kobayashi SD, Whitney AR, Voyich JM, Argue CM, et al (2005) Insights into pathogen immune evasion mechanisms: Anaplasma phagocytophilum fails to induce an apoptosis differentiation program in human neutrophils. J Immunol 174: 6364-6372.

28. Woldehiwet $Z$ (2008) Immune evasion and immunosuppression by Anaplasma phagocytophilum, the causative agent of tick-borne fever of ruminants and human granulocytic anaplasmosis. Vet J 175: 37-44.

29. Ballesteros C, Garrido JM, Vicente J, Romero B, Galindo RC, et al. (2009) First data on Eurasian wild boar response to oral immunization with BCG and challenge with a Mycobacterium bovis field strain. Vaccine 27: 6662-6668.
Submit your next manuscript and get advantages of OMICS Group submissions

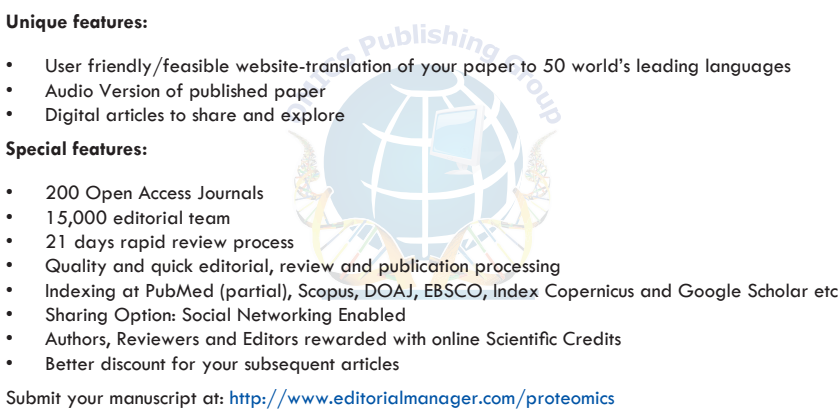

